\title{
乌ु
}

\section{Pressure Induced Superconductivity on the border of Magnetic Order in MnP}

\author{
J.-G. Cheng, ${ }^{1,2,{ }^{*}}$ K. Matsubayashi, ${ }^{2}$ W. Wu, ${ }^{1}$ J. P. Sun, ${ }^{1}$ F. K. Lin, ${ }^{1}$ J. L. Luo, ${ }^{1,3}$ and Y. Uwatoko ${ }^{2}$ \\ ${ }^{1}$ Beijing National Laboratory for Condensed Matter Physics and Institute of Physics, \\ Chinese Academy of Sciences, Beijing 100190, China \\ ${ }^{2}$ Institute for Solid State Physics, University of Tokyo, 5-1-5 Kashiwanoha, Kashiwa, Chiba 277-8581, Japan \\ ${ }^{3}$ Collaborative Innovation Center of Quantum Matter, Beijing, China
}

(Received 17 December 2014; published 16 March 2015)

\begin{abstract}
We report the discovery of superconductivity on the border of long-range magnetic order in the itinerantelectron helimagnet $\mathrm{MnP}$ via the application of high pressure. Superconductivity with $T_{\mathrm{sc}} \approx 1 \mathrm{~K}$ emerges and exists merely near the critical pressure $P_{c} \approx 8 \mathrm{GPa}$, where the long-range magnetic order just vanishes. The present finding makes MnP the first Mn-based superconductor. The close proximity of superconductivity to a magnetic instability suggests an unconventional pairing mechanism. Moreover, the detailed analysis of the normal-state transport properties evidenced non-Fermi-liquid behavior and the dramatic enhancement of the quasiparticle effective mass near $P_{c}$ associated with the magnetic quantum fluctuations.
\end{abstract}

Extensive investigations over the last decade have uncovered the quantum criticality as a universal phenomenon connecting with many difficult problems in modern physics [1,2]. For example, the most distinguished problem of unconventional superconductivity (SC) as found in several distinct superconducting systems including the heavy-fermion, organic, cuprates, and the iron-based superconductors can be generally described in the framework of the antiferromagnetic quantum critical point (QCP) [3-6]. The close proximity of $\mathrm{SC}$ to a magnetic instability suggests that the critical spin fluctuations would play a crucial role for mediating the Cooper pairs [5,7]. On the other hand, to realize a magnetic QCP should provide an effective approach for searching new classes of unconventional superconductors. This is well illustrated by the recent discovery of pressure-induced SC in CrAs [8,9], the first Cr-based unconventional superconductor [10]. This discovery has left manganese (Mn) the only $3 d$ element that does not show SC among any Mn-based compounds, even though a great effort has been devoted recently to explore the possible SC via carrier doping [11] or the application of high pressure [12]. The strong magnetism of $\mathrm{Mn}$ is commonly believed to be antagonistic to SC. Therefore, it is highly interesting to explore whether SC can emerge near a magnetic QCP in the Mn-based compounds.

The itinerant-electron helimagnet, MnP [13], with a much reduced moment of $\sim 1.3 \mu_{B} / \mathrm{Mn}$ has attracted our attention as a good starting point to approach a magnetic instability. At ambient condition, $\mathrm{MnP}$ adopts an orthorhombic B31-type structure with lattice constants $a=5.26$, $b=3.17$, and $c=5.92 \AA$, respectively [14]. In the absence of a magnetic field, MnP undergoes two successive magnetic transitions upon cooling: [13] a transition from the paramagnetic (PM) to ferromagnetic (FM) state at $T_{C}=291 \mathrm{~K}$, and then a second transition to a double helical state at $T_{s} \approx 50 \mathrm{~K}$. In the FM state, the Mn spins are aligned parallel to the orthorhombic $b$ axis, and the ordered moment is about $1.3 \mu_{B} / \mathrm{Mn}$. In the double helical state, the $\mathrm{Mn}$ spins rotate in the $a b$ plane with the propagation vector $\mathbf{q}$ along the $c$ axis [15]. Earlier hydrostatic pressure studies [16-18] on MnP have revealed that both $T_{s}$ and $T_{C}$ decrease with pressure. However, the pressure and temperature ranges in the previous studies were far from the magnetic QCP. By utilizing the recently developed highpressure techniques [19-23], we have reinvestigated this problem in an extended pressure and temperature range, and surprisingly found that $\mathrm{MnP}$ becomes superconducting below $T_{\mathrm{sc}} \approx 1 \mathrm{~K}$ near the magnetic QCP at $P_{c} \approx 8 \mathrm{GPa}$. Since the majority of the density of states near the Fermi level for $\mathrm{MnP}$ is attributed to the $\mathrm{Mn}-3 d$ states [24,25], the present discovery makes $\mathrm{MnP}$ the first $\mathrm{Mn}$-based superconductor, and the close proximity of SC to a magnetic instability suggests an unconventional pairing mechanism.

Needle-shaped MnP single crystals used in the present study were grown out of a Sn flux. Measurements of resistivity $\rho(T)$ and ac magnetic susceptibility $\chi^{\prime}(T)$ under pressures up to $10 \mathrm{GPa}$ were performed by using various high-pressure techniques. Without specification, the resistivity in the present study was measured with the current applied along the orthorhombic $b$ axis, the easymagnetization direction. Details about the crystal growth and the high-pressure measurements can be found in the Supplemental Material [26].

Figure 1(a) shows the $b$-axis $\rho(T)$ data under various pressures up to $10.7 \mathrm{GPa}$ measured with a palm cubic anvil cell. In agreement with the previous reports [27], $\rho(T)$ at zero pressure display a kink anomaly at the FM transition, $T_{C}=291 \mathrm{~K}$, which can be defined clearly as a sharp peak in the $d \rho / d T$ curve, Fig. 1(b). The $\rho(T)$ and $d \rho / d T$ curves at 2.8 GPa keep essentially similar features as those at 

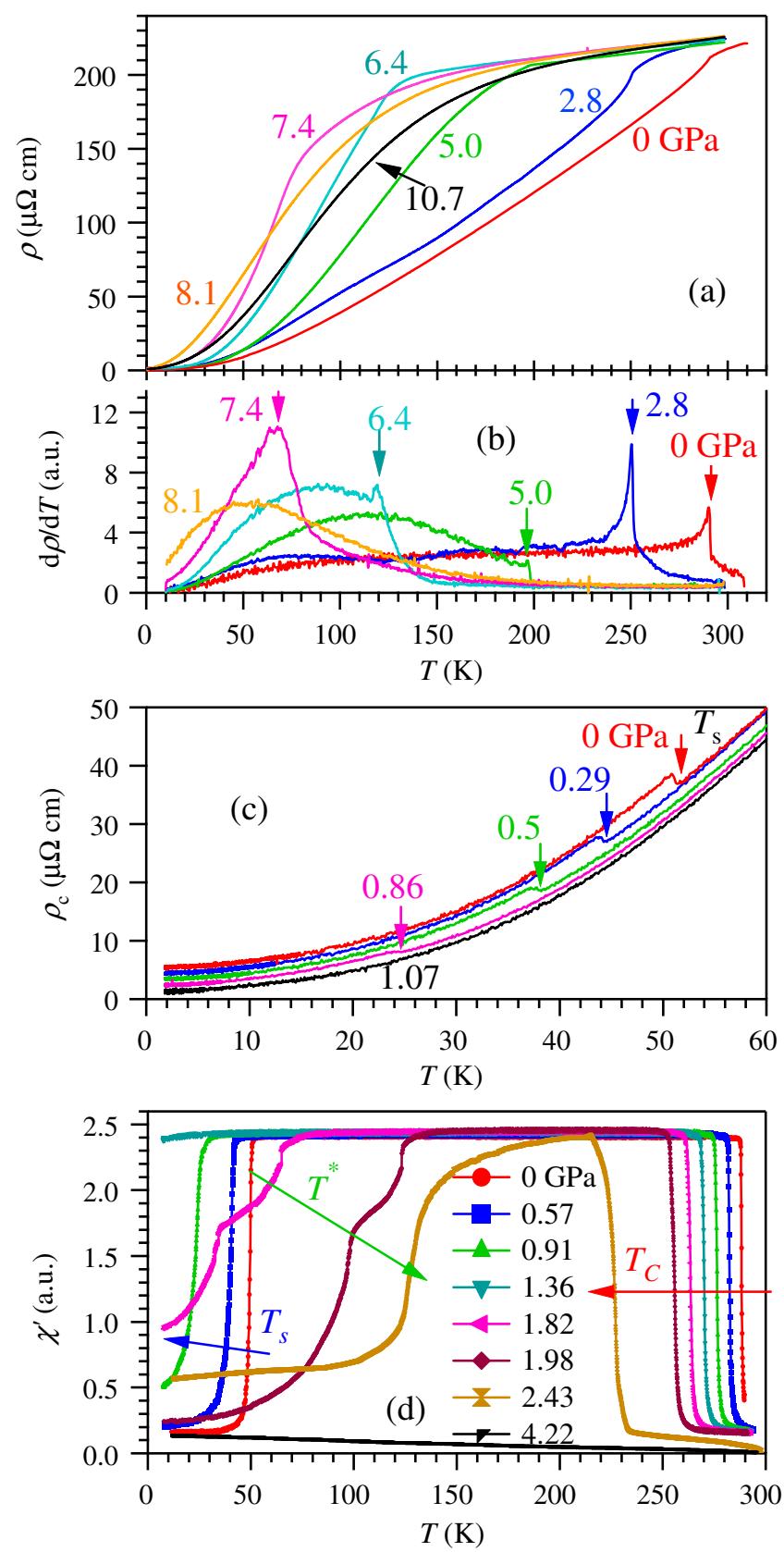

FIG. 1 (color online). (a) Resistivity $\rho(T)$ and (b) the temperature derivative $d \rho / d T$ of the MnP single crystal under various pressures up to $10.7 \mathrm{GPa}$ highlighting the variation with pressure of the magnetic transition indicated by the vertical arrows. (c) The $c$-axis $\rho_{c}(T)$ data at low temperatures highlighting the evolution with pressure of the double helical transition at $T_{s}$. (d) Temperature dependence of the ac magnetic susceptibility $\chi^{\prime}(T)$ measured under various pressures, which evidences the appearance of a new magnetic transition at $T^{*}$.

ambient pressure, except that $T_{C}$ has been shifted down to $\sim 250 \mathrm{~K}$. Upon further increasing pressure to $5.0 \mathrm{GPa}$, however, the temperature profile of $\rho(T)$ exhibits distinct features with a clear inflection point at $\sim 200 \mathrm{~K}$, which corresponds to a steplike anomaly in the $d \rho / d T$ curve in
Fig. 1(b). As noticed by Banus [18] and discussed below, it is most probable that the FM transition changes to an antiferromagnetic (AFM) type for $P>3 \mathrm{GPa}$. For this reason, we label the transition temperature as $T_{m}$ for $P>$ $3 \mathrm{GPa}$ hereafter. The $\rho(T)$ and $d \rho / d T$ curves at $6.4 \mathrm{GPa}$ are similar to that at $5.0 \mathrm{GPa}$, except that the magnetic transition broadens up and moves down to $\sim 120 \mathrm{~K}$. Upon further increasing pressure to $7.4 \mathrm{GPa}$, nevertheless, $\rho(T)$ changes again with the concave curvature restored for $T>T_{m}=70 \mathrm{~K}$, which is manifested as a relatively broad peak in the $d \rho / d T$ curve in Fig. 1(b). Above this pressure, no clear anomaly can be discerned in the $\rho(T)$ curves. In contrast with the complete suppression of the magnetic transition in the present work, an earlier high-pressure study [18] found that the magnetic transition temperature is almost pressure independent or even slightly increases with pressure for $3<P<5 \mathrm{GPa}$ when using the solid $(\mathrm{AgCl})$ pressure transmitting medium. This comparison suggests that the magnetic transition of $\mathrm{MnP}$ is very sensitive to the pressure conditions; the nonhydrostatic pressure would prevent the realization of the magnetic QCP and the SC shown below.

Figure 1(c) shows the $c$-axis resistivity $\rho_{c}(T)$ measured in a piston-cylinder cell. As reported earlier [27], $\rho_{c}(T)$ at ambient pressure exhibits a clear dip anomaly at $T_{s}$ because the helical magnetic structure propagates along the $c$ axis. As shown clearly in Fig. 1(c), $T_{s}$ decreases monotonically with pressure and vanishes completely at $\sim 1 \mathrm{GPa}$.

We also followed directly the evolution with pressure of the magnetic transitions at $T_{C}$ and $T_{s}$ with the $\chi^{\prime}(T)$ under pressure. As shown in Fig. 1(d), they are manifested as a sudden jump and drop, respectively, at ambient pressure, and the FM state corresponds to the in-between plateau. In agreement with the $\rho(T)$ data, $T_{C}$ decreases continuously, and $T_{s}$ vanishes completely around $1.4 \mathrm{GPa}$. Surprisingly, a new two-step transition denoted as $T^{*}$ emerges above $1.4 \mathrm{GPa}$ and increases quickly with pressure. Since no anomaly in $\chi^{\prime}(T)$ can be discerned at $4.2 \mathrm{GPa}$, the magnetic transitions reflected in $\rho(T)$ above $3 \mathrm{GPa}$ should correspond to an AFM one. However, whether this AFM state is similar with the low-pressure double helical phase at $T<T_{s}$ deserves further studies.

From the above results, we can see that the application of high pressure quickly eliminates the double helical state, and first reduces the FM transition at $T_{C}$, and then changes it to an AFM type for $P>3 \mathrm{GPa}$. The magnetic transition monitored by the anomaly in $\rho(T)$ eventually vanishes completely around $P_{c} \approx 8 \mathrm{GPa}$, where the most striking change takes place at low temperatures. As shown in Fig. 2(a), we start to see a resistivity drop below $1 \mathrm{~K}$ at 7.6 $\mathrm{GPa}$, and a more pronounced drop with an onset temperature of $\sim 1 \mathrm{~K}$ is clearly observed near the critical pressure $P_{c} \approx 7.8 \mathrm{GPa}$, which signals the possible occurrence of SC. With further increasing pressure, this anomaly shifts to lower temperatures. Although zero resistivity can 


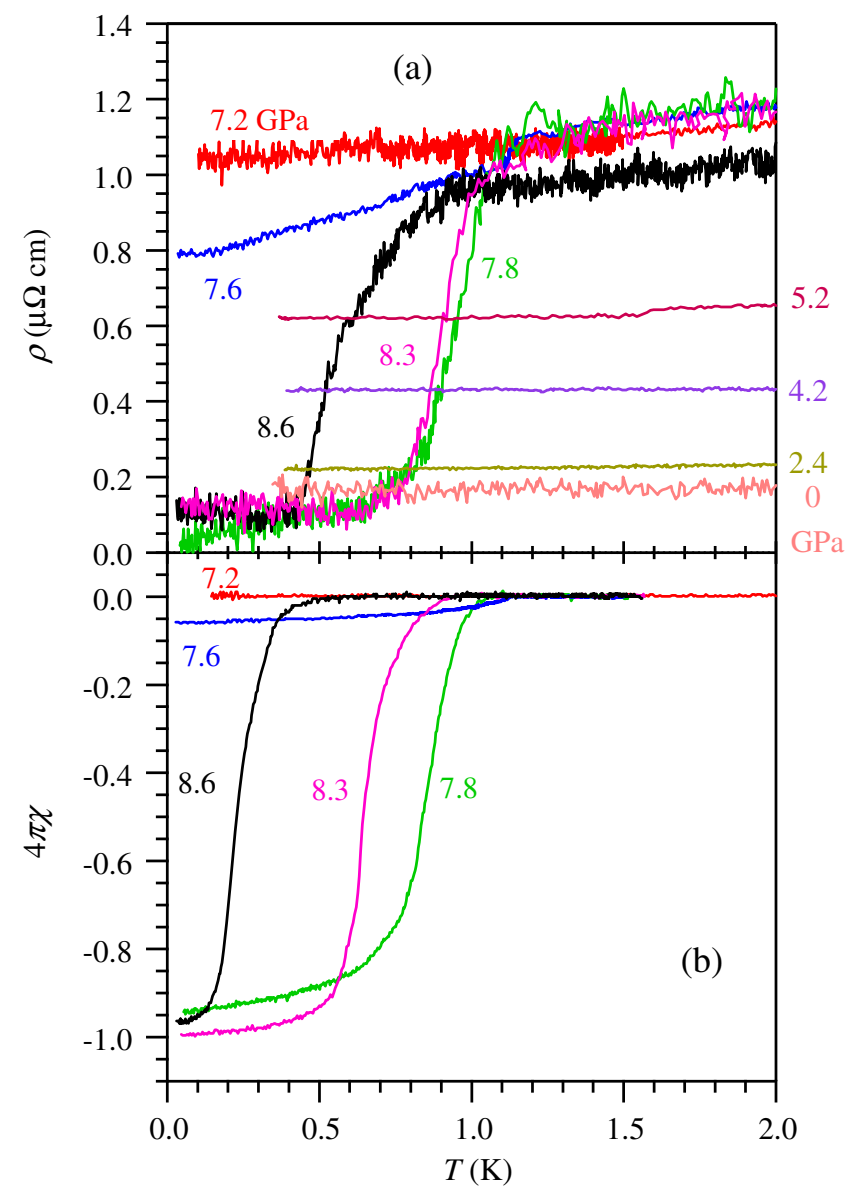

FIG. 2 (color online). Temperature dependence of (a) the resistivity $\rho(T)$ and (b) the ac magnetic susceptibility $4 \pi \chi$ on $\mathrm{MnP}$ at low temperatures under various pressures near the critical pressure.

be hardly reached even when the applied electrical current is reduced to $10 \mu \mathrm{A}$, the ac magnetic susceptibility, $4 \pi \chi(T)$, shown in Fig. 2(b) provides strong evidence for the occurrence of SC near $P_{c}$. In perfect agreement with the $\rho(T)$ data, the diamagnetic signal appears below $T_{\mathrm{sc}} \approx 1 \mathrm{~K}$ at $7.6 \mathrm{GPa}$, and the superconducting shielding fraction reaches $\sim 95 \%$ of the sample volume at $7.8 \mathrm{GPa}$. A further increment of pressure to $8.6 \mathrm{GPa}$ lowers $T_{\mathrm{sc}}$ to below $0.5 \mathrm{~K}$, while the superconducting shielding fraction keeps nearly constant. Such a perfect diamagnetic response rules out the possibility of filamentary SC or impurity phases. However, the absence of zero resistivity could be caused by the imperfect sample quality or the pressure inhomogeneity [28]. The observation that the SC disappears quickly after the magnetic transition vanishes completely highlights that the pressure-induced SC has an intimate correlation with the magnetic critical point.

To gain further insights into the superconducting state, we obtained the upper critical field $\mu_{0} H_{\mathrm{c} 2}$ from the field dependence of $\rho(T)$ and $4 \pi \chi(T)$ at $P=7.8 \mathrm{GPa}$, Figs. 3(a) and 3(b), and plotted $\mu_{0} H_{\mathrm{c} 2}$ as a function of $T_{\mathrm{sc}}$ in Fig. 3(c). Here, we define $T_{\mathrm{sc}}$ as the temperatures corresponding
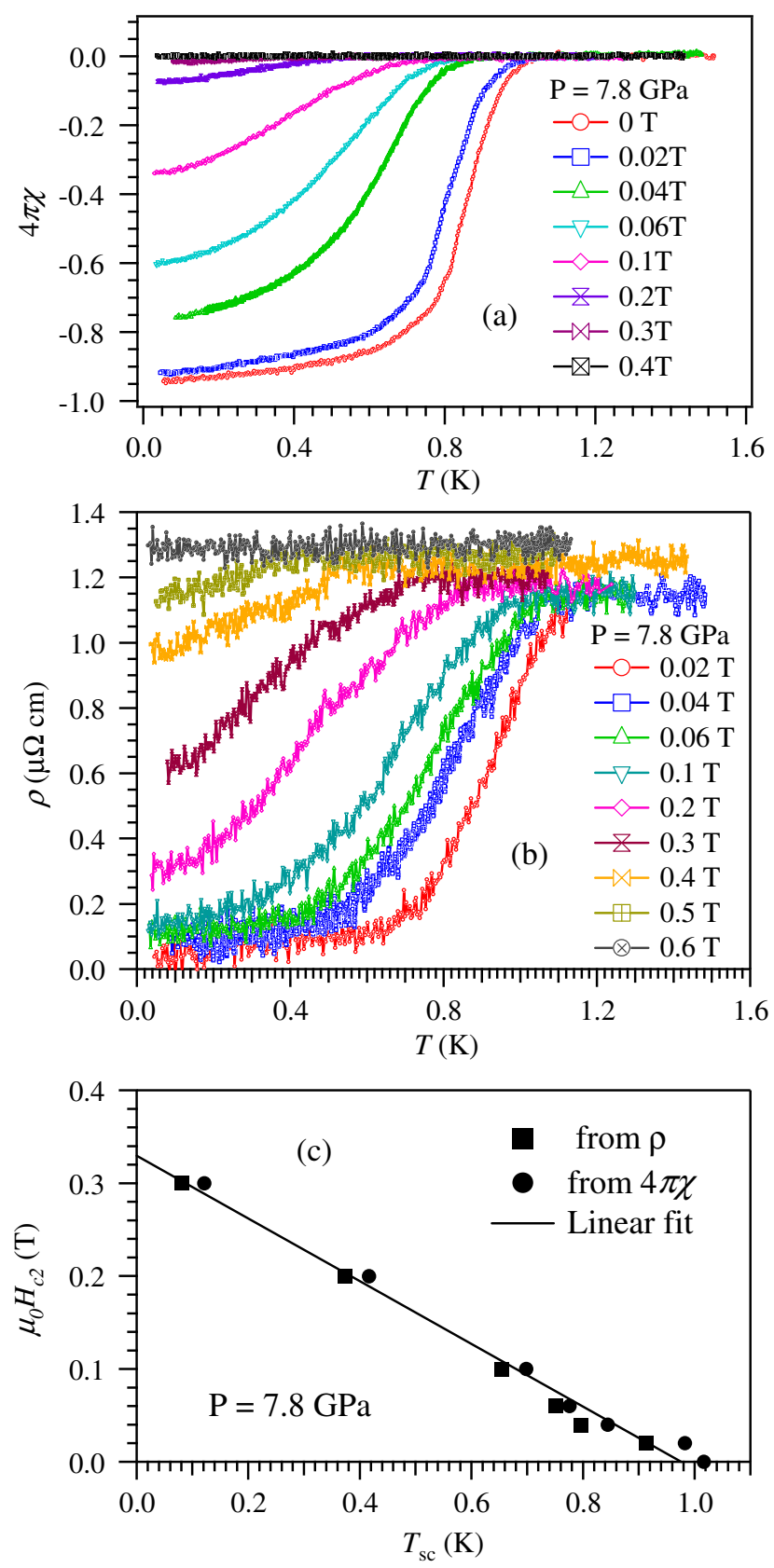

FIG. 3 (color online). Temperature dependence of (a) ac magnetic susceptibility $4 \pi \chi$ and (b) resistivity $\rho(T)$ under different magnetic fields at 7.8 GPa. (c) Temperature dependence of the upper critical field $\mu_{0} H_{\mathrm{c} 2}$ for $\mathrm{MnP}$ at $7.8 \mathrm{GPa}$. The solid line in (c) is a linear fitting, which gives $\mu_{0} H_{\mathrm{c} 2}(0)=0.33(1) \mathrm{T}$, and a slope $-0.34(1) \mathrm{T} / \mathrm{K}$.

to a $50 \%$ resistivity drop and a $1 \%$ ac susceptibility drop. As shown in Fig. 3(c), $\mu_{0} H_{\mathrm{c} 2}$ versus $T_{\mathrm{sc}}$ is better described by a linear fitting, which yields a $\mu_{0} H_{\mathrm{c} 2}(0)=0.33(1) \mathrm{T}$, and an initial slope of $-\mu_{0} d H_{\mathrm{c} 2} /\left.d T_{\mathrm{sc}}\right|_{T s c}=0.34(1) \mathrm{T} / \mathrm{K}$. The obtained $\mu_{0} H_{\mathrm{c} 2}(0)$ allows us to estimate the Ginzburg-Landau coherence length $\xi=315 \AA$ according to the relationship: $\mu_{0} H_{\mathrm{c} 2}(0)=\Phi_{0} / 2 \pi \xi^{2}$, where $\Phi_{0}=$ $2.067 \times 10^{-15} \mathrm{~Wb}$ is the magnetic flux quantum [29]. 
(a)
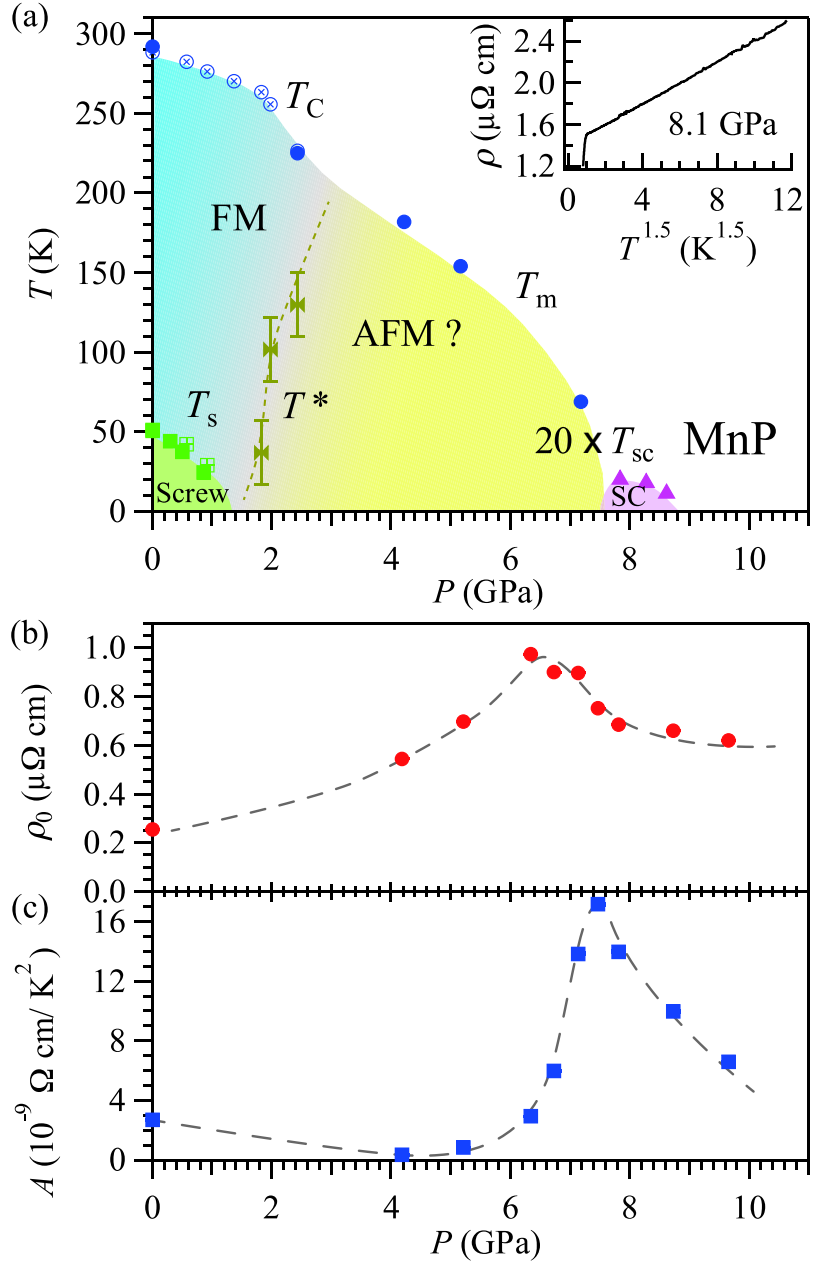

FIG. 4 (color online). (a) Pressure dependences of the magnetic transition temperatures, $T_{C}, T_{m}, T^{*}, T_{s}$, and the superconducting transition temperature $T_{s c} ; T_{\mathrm{sc}}$ has been scaled by a factor of 20 for clarity. (b),(c) Variation with pressure of the residual resistivity $\rho_{0}$ and the $A$ coefficient extracted from a linear fitting to the $\rho\left(T^{2}\right)$ curves at the low-temperature limit. Inset of (a) shows the low-temperature resistivity data at $8.1 \mathrm{GPa}$ in the form of $\rho$ versus $T^{1.5}$. The relatively large error bar for $T^{*}$ in (a) reflects the two-step feature evidenced in the ac magnetic susceptibility in Fig. 1(d).

The transition temperatures, $T_{C}, T_{m}, T_{s}, T^{*}$, and $T_{s c}$, obtained from the above measurements are mapped into the temperature-pressure phase diagram shown in Fig. 4(a). As can be seen clearly, the application of high pressure reduces continuously the magnetic transition temperatures, $T_{C}$ and then $T_{m}$, and eventually suppresses the magnetic order around $P_{c} \approx 8 \mathrm{GPa}$. Superconductivity with a maximum $T_{\mathrm{sc}} \approx 1 \mathrm{~K}$ emerges and exists within a narrow pressure range near the critical pressure $P_{c}$. Such a superconducting $T-P$ phase diagram is remarkably similar with that of heavy-fermion superconductors, such as $\mathrm{CeIn}_{3}$ and $\mathrm{CePd}_{2} \mathrm{Si}_{2}$, [3] in which a magnetically mediated mechanism is believed to play a dominant role for forming Cooper pairs. In the case of $\mathrm{MnP}$, the situation becomes more complicated in that the application of pressure alters the nature of the FM transition to an AFM type around $3 \mathrm{GPa}$. Although the nature of the AFM state remains elusive, we provide below some evidences that are in favor of an antiferromagnetic QCP at $P_{c}$, and thus an unconventional pairing mechanism for the observed SC in MnP.

As shown in the inset of Fig. 4(a), the low-temperature normal-state resistivity near $P_{c}$ exhibits a non-Fermi-liquid behavior $\rho(T) \propto T^{n}$ with $n \approx 1.5$, consistent with the theoretical prediction for the incoherent scattering of quasiparticles near a 3D antiferromagnetic QCP [3]. In addition, a linear fitting to the $\rho\left(T^{2}\right)$ curves (Fig. S4), i.e., $\rho=\rho_{0}+A T^{2}$, in the low-temperature limit evidenced significant enhancements of both the residual resistivity $\rho_{0}$ and the $A$ coefficient near $P_{c}$, Figs. 4(b) and 4(c). Since the $A$ coefficient is proportional to the effective mass of charge carriers via $A \propto\left(m^{*} / m_{0}\right)^{2}$, the significant enhancement of $A$ signals a dramatic enhancement of effective mass associated with the suppression of magnetic order. These above observations near $P_{c}$ have been regarded as characteristic signatures of an antiferromagnetic QCP in strongly correlated metallic systems, and provide important clues for the unconventional nature of the pressure-induced SC in MnP.

Finally, it is interesting to mention another itinerantelectron helimagnet $\mathrm{MnSi}$, which has been extensively studied in the context of a ferromagnetic QCP [30]. Although anomalous metallic properties have been observed near the QCP, no SC has been observed down to very low temperatures. This comparison highlights the importance of the antiferromagnetic spin fluctuations associated with a QCP for the observed SC in MnP. The mechanism of pressure-induced ferromagnetic-to-antiferromagnetic transition in MnP thus deserves further studies. Goodenough has argued that such a pressure-induced ferromagnetic-to-antiferromagnetic transition is due to bandwidth broadening in the narrow $d$-band system [18,31].

In summary, we have found that the itinerant helimagnet MnP becomes superconducting below $T_{\mathrm{sc}} \approx 1 \mathrm{~K}$ when its long-range magnetic order is completely suppressed by the application of high pressure around $P_{c} \approx 8 \mathrm{GPa}$. The close proximity of SC to a magnetic instability suggests an unconventional pairing mechanism. The present finding of the first Mn-based superconductor breaks the general wisdom about the Mn's antagonism to superconductivity. We hope that this discovery will stimulate more work on searching for Mn-and other transition-metal-based superconductors with a higher transition temperature.

We thank Y. P. Wang, J. Q. Yan, J.S. Zhou, N. Mori, F. Steglich, Q. Si, P. J. Sun, P. Dai, and P. Coleman for enlightening discussions. We are also grateful to $\mathrm{S}$. Nagasaki and K. Kitagawa for their technical support. Work at IOP/CAS was supported by the National Basic Research Program of China (Grants No. 2014CB921500, No. 2011CB921700, No. 2015CB921300), the National Science Foundation of China (Grants No. 11304371, 
No. 11025422), and the Strategic Priority Research Program (B) of the Chinese Academy of Sciences (Grants No. XDB07020100, No. XDB01020300). Work at ISSP/UT was partially supported by Grant-in-Aid for Scientific Research, KAKENHI (Grants No. 23340101, No. 252460135), and the JSPS fellowship for foreign researchers (Grant No. 12F02023).

J.-G. C., K. M., and W. W. contributed equally to this work.

*jgcheng@iphy.ac.cn

[1] P. Coleman and A. J. Schofield, Nature (London) 433, 226 (2005).

[2] S. Sachdev and B. Keimer, Phys. Today 64, 29 (2011).

[3] N. D. Mathur, F. M. Grosche, S. R. Julian, I. R. Walker, D. M. Freye, R. K. W. Haselwimmer, and G. G. Lonzarich, Nature (London) 394, 39 (1998).

[4] M. R. Norman, Science 332, 196 (2011).

[5] P. Monthoux, D. Pines, and G. G. Lonzarich, Nature (London) 450, 1177 (2007).

[6] P. Gegenwart, Q. Si, and F. Steglich, Nat. Phys. 4, 186 (2008).

[7] O. Stockert et al., Nat. Phys. 7, 119 (2011).

[8] W. W. J.-G. Cheng, K. Matsubayashi, P. P. Kong, F. K. Lin, C. Q. Jin, N. L. Wang, Y. Uwatoko, and J. L. Luo, Nat. Commun. 5, 5508 (2014).

[9] H. Kotegawa, S. Nakahara, H. Tou, and H. Sugawara, J. Phys. Soc. Jpn. 83, 093702 (2014).

[10] H. Kotegawa, H. Tou, H. Sugawara, and H. Harima, arXiv:1408.3185.

[11] J. W. Simonson et al., Proc. Natl. Acad. Sci. U.S.A. 109, E1815 (2012).

[12] J. Guo et al., Sci. Rep. 3, 2555 (2013).

[13] E. E. J. Huber and H. D. Ridgley, Phys. Rev. 135, A1033 (1964).

[14] Here we use the Pnma setting $c>a>b$ through this Letter.
[15] G. P. Felcher, J. Appl. Phys. 37, 1056 (1966).

[16] T. Kamigaich, T. Okamoto, N. Iwata, and E. Tatsumoto, J. Phys. Soc. Jpn. 24, 649 (1968).

[17] N. P. Grazhdankina, A. M. Burkhanov, and Y. S. Bersenev, Sov. Phys. JETP 28, 1141 (1969).

[18] M. D. Banus, J. Solid State Chem. 4, 391 (1972).

[19] Y. Uwatoko, Rev. High Pres. Sci. Tech. 12, 306 (2002).

[20] Y. Uwatoko et al., Rev. High Pres. Sci. Tech. 18, 230 (2008).

[21] K. Matsubayashi, A. Hisada, T. Kawae, and Y. Uwatoko, Rev. High Pres. Sci. Tech. 22, 206 (2012).

[22] J.-G. Cheng, K. Matsubayashi, S. Nagasaki, A. Hisada, T. Hirayama, M. Hedo, H. Kagi, and Y. Uwatoko, Rev. Sci. Instrum. 85, 093907 (2014).

[23] K. Kitagawa, H. Gotou, T. Yagi, A. Yamada, T. Matsumoto, Y. Uwatoko, and M. Takigawa, J. Phys. Soc. Jpn. 79, 024001 (2010).

[24] A. Yanase and A. Hasegawa, J. Phys. C 13, 1989 (1980).

[25] P. G. Perkins, A. K. Marwaha, and J. J. P. Stewart, Theoret. Chim. Acta (Berl.) 59, 569 (1981).

[26] See Supplemental Material at http://link.aps.org/ supplemental/10.1103/PhysRevLett.114.117001 for the experimental details and the supporting results, which includes Refs. [19-23].

[27] A. Takase and T. Kasuya, J. Phys. Soc. Jpn. 48, 430 (1980).

[28] Considering the narrow pressure range where superconductivity exists, a slight pressure distribution or inhomogeneity could influence the superconducting state of MnP.

[29] P. G. de Gennes, Superconductivity of Metals and Alloys (Benjamin, New York, 1966).

[30] N. Doiron-Leyraud, I. R. Walker, L. Taillefer, M. J. Steiner, S. R. Julian, and G. G. Lonzarich, Nature (London) 425, 595 (2003).

[31] J. B. Goodenough, in Progress in Solid State Chemistry, edited by H. Reiss (Pergamon Press, Oxford, 1971), Chap. 4. 